\title{
Cross-layer improvement for TCP Westwood and VoIP over satellite
}

\author{
M.Kalama ${ }^{1,2}$, M-P.Kluth ${ }^{1}$, S.Mourgues ${ }^{1}$, B.Evans ${ }^{2}$ \\ ${ }^{1}$ Centre for Communication Systems Research, University of Surrey, Guildford, GU27XH, UK \\ ${ }^{2}$ EADS ASTRIUM, 31 Rue des Cosmonautes, Toulouse, 31402, France \\ m.kalama $a$ surrey.ac.uk \\ marie-paule.kluth@astrium.eads.net \\ stephane.mourgues $a$ astrium.eads.net \\ b.evans@surrey.ac.uk
}

\begin{abstract}
Satellite communications can play an important role in extending the reach of broadband networks to terrestrially underserved areas. However, several popular internet applications could suffer from performance degradation over satellite (e.g. realtime gamming, web browsing, VoIP), thus necessitating protocol modifications and additional optimisation mechanisms. Cross-layer design, which is the optimisation technique adopted in this paper, is a relatively new idea in network design. It is based on blurred boundaries between network layers. In particular, two cross-layer mechanisms are described; the first adapts the VoIP speech coding rate according to network congestion while the second uses TCP state information at MAC layer in order to improve the throughput of TCP traffic. Analysis in this paper demonstrates that the use of cross-layer techniques could improve the performance of the targeted applications in terms of reduced delay while continuously maintaining an optimal speech coding rate for VoIP.
\end{abstract}

\section{INTRODUCTION}

Unlike the separate optimization of individual layers within the protocol stack, the cross-layer design concept assumes that mechanisms in a particular layer can be improved by making use of the state information from any other layer.

The aim of this paper is to illustrate the concept of crosslayer design in the context of satellite broadband networks while applying it for the improvement of resource management. The two cross-layer mechanisms described, aim to improve the flexibility as well as responsiveness of the underlying DAMA (Demand Assignment Multiple Access) scheme that is used to share the MF-TDMA satellite uplink capacity, based on appropriate information from other layers.

The software tool to be used for the simulations is also presented in the paper. It is developed by integrating an EADS ASTIUM MAC-layer simulator with ns-2, a popular open source network simulation tool [17]. This flexible, hybrid simulator allows integrating a large variety of available higher layer protocol modules developed for ns, and analysing their performances over a comprehensive and accurate implementation of the DVB-S2/DVB-RCS air-interface.
This research work is based on the ongoing GesAReS ${ }^{1}$ study (Adaptive Management of Satellite Resources in Broadband Systems in $\mathrm{Ka}$ and $\mathrm{Q} / \mathrm{V}$ bands using Fade Mitigation Techniques) as well as the activities during the first year of the author's PhD study, which is supported by EADS Astrium. GesAReS is funded by CNES, primed by EADS Astrium and carried out in co-operation with INT (Institut National des Telecommunication).

The rest of the paper is organised in five sections. Section II describes the reference satellite system based on which the crosslayer solutions will be assessed. Section III briefly presents the performance issues addressed. The proposed VoIP cross-layer mechanism is described in Section IV. The second cross-layer algorithm is then presented in Section V and section VI presented the ns/SSP simulator. Finally, in section VII, our conclusions are presented together with a discussion of the future activities planned in this study.

\section{SYstem ARCHITECTURE SCENARTO}

The considered reference architecture is compliant with the DVB-S2/DVB-RCS standards [18], [19]. It includes gateways connected to the Internet, satellite terminals (RCSTs) hosted at end-user premises, a geostationary transparent satellite and a network control center (NCC). The NCC is connected to all GWs and RCSTs (via GWs) and is responsible for the overall system configuration and supervision.

GWs also implement link control and some management functionalities as well as DiffServ marking and TCP Performance Enhancement Proxies (PEP) for the optimisation of TCP connections.

Quality of Service (QoS) is supported through three DiffServ traffic queues (Expedited Forwarding EF; Assured Forwarding $\mathrm{AF}$ and Best Effort BE) and associated DVB-RCS capacity categories. Each RCST interfaces with a given GW and contributes to resource management through the transmission of connection requests and dynamic capacity requests (RBDC and

\footnotetext{
${ }^{1}$ Gestion Adaptative des Ressources Satellitaires dans les systèmes large bande dans les bandes $\mathrm{Ka}$ et $\mathrm{Q} / \mathrm{V}$ utilisant des techniques de fade mitigation
} 
(A)VBDC) but also through their involvement in link control. Then symmetrically to GWs they also include management functionalities, DiffServ marking and TCP PEPs.

Regarding Fade Mitigation Techniques (FMT) support, the reference architecture implements Adaptive Coding and Modulation (ACM) on the forward link and Adaptive Coding + Dynamic Rate Adaptation (AC + DRA) on the return link. The FMT algorithms, developed by EADS ASTRIUM, are modeled in the SSP software allowing an analysis of bandwidth efficiency as well as BER.

\section{Cross-layer design AND SAtellite networks}

\section{A. Cross-layer design}

Cross-layer design first appeared in the context of wireless and more specifically ad-hoc networks and since then the concept has spread into research for most network domains. Work such as [1] or [2] offers a useful classification of crosslayer mechanisms based on the direction of information flow within the protocol stack.

In the satellite research domain not as many publications existed until recently specifically addressing cross layer design, despite the satellite environment being highly dynamic both in terms of the physical wireless channel but also due to the dynamic capacity management. [3], [4], [5] and [6] constitute examples of recent work. However, similar issues have been addressed in literature, even if not labeled as cross-layer design as such, concerning adaptive PEP congestion strategies, MAC layer resource management techniques combined with TCP or mapping Diffserv CoS to DVB-RCS capacity categories.

\section{B. Satellite VoIP and Bandwidth on Demand}

As there are numerous possibilities for information exchange within the protocol stack, work in this paper has been targeted towards the optimization of two application scenario types, VoIP as a real-time UDP application and TCP applications with near real-time requirements such as (V/AoD) over TCP.

VoIP over satellite is mainly affected by the large propagation delay and jitter. To address these issues, work by ground segment vendors ([7], [8], [9], and [10]) or industrial groups such as SatLabs have proposed suitable QoS techniques (i.e. DiffServ) as well as flexible BoD (Bandwidth on Demand) schemes for the return link. In addition, advances in header and voice compression, smart packetisation and silence suppression improve capacity utilisation.

The newly introduced FMT techniques result in a reduction of the useful bandwidth, when robust coding is applied. This reduction can lead potentially to congestion and higher layer transport or application protocols have to adapt to it. However, for real time services this can be difficult due to the connectionless nature of UDP. Cross-layer design may assist therefore in improving this interaction between different layers in aligning metrics such as the application and physical layer information rates.

For TCP applications, the use of performance Enhancing Proxies already improves several of the widely known TCP impairments over satellite. However, return link BoD schemes ensure indeed flexible capacity management and low delay for priority applications but to the expense of service interactivity and delay for AF or BE TCP applications, which need to go through the requesting process of non guaranteed bandwidth.

\section{THE ADAPTIVE RATE VOIP ALGORITHM}

\section{A. The symmetric transcoding scenario}

Based on the algorithm proposed in [17], the Adaptive rate VoIP mechanism aims to adapt the speech coding rate of VoIP sessions according to network congestion. The process involves transcoders both for the forward and return links at the edges of the satellite network (i.e. GW and RCST) in order to keep the process transparent from end users. The reduction of the end user speech coding rate by the transcoder is performed in response to potential delay caused either by bad atmospheric conditions (resulting in robust coding) or by increased traffic load.

The steps of the algorithm are presented below for the RCST side. It should be noted here that the transcoder has the following nine coding modes: rate $[$ mode $]=[6.6,8.85,12.65,14.25,15.85$, $18.25,19.25,23.05,23.85] \mathrm{kbps}$

Step 1: Detect data flow via the transcoder and call the function to adjust rate.

Step 2a: Set up the initial coding rate according to the current mean rate of the EF queue due to concurrent traffic calculated by (1):

$$
\eta_{\text {mean }}=\eta(t-1)^{*} \alpha+(1-\alpha)^{*} \eta(t)
$$

where $\eta_{\text {mean }}$ is the EF queue mean rate, $\eta(t)$ is the average EF output rate measured at the RCST queue over a period of $x$ (typically 3-4) superframes and the parameter $\alpha$ is initially set to 0.1 .

Accordingly, the available mean rate for the VoIP flow is:

$$
\text { maximum mean rate - current mean rate }
$$

where the maximum mean rate is defined in the SLA (Service Level Agreement) of the user as the sum of the CRA plus minimum RBDC capacity.

Step 2b: Continue monitoring the $\mathrm{EF}$ queue output rate and in case of congestion reduce the coding rate by an equivalent amount to the congestion.

Step 3: Terminate the process when flow of data via the transcoder has stopped.

As the mechanism is symmetric, the same steps are also followed for the forward path transcoding process using equivalent data from the GW. 


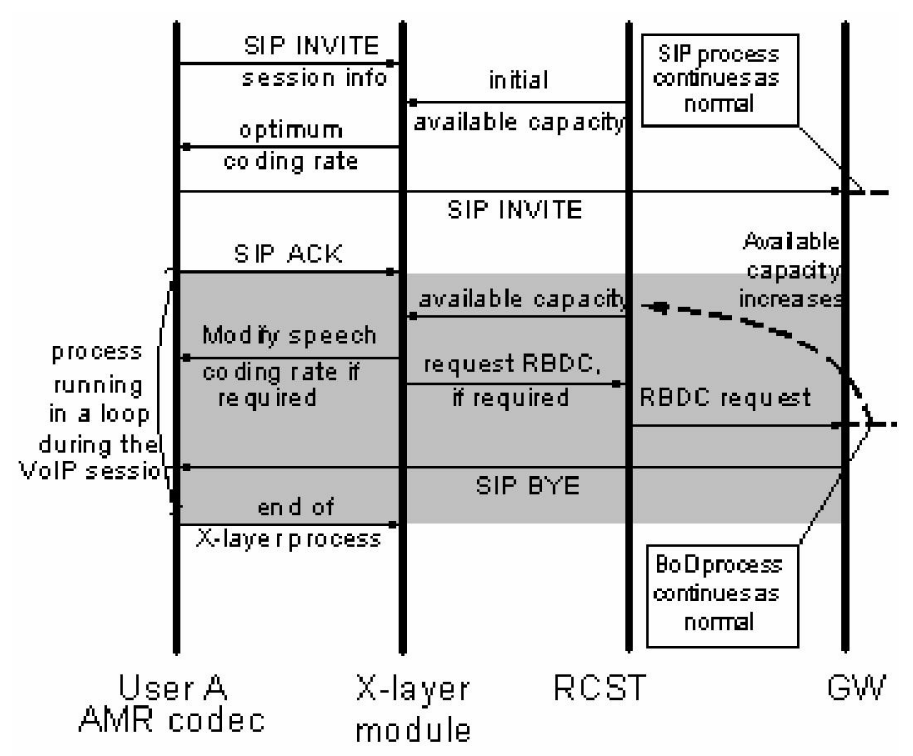

Fig. 1. Illustration of the adaptive multi-rate codec scenario

\section{B. The adaptive multi-rate codec scenario}

The second scenario of the mechanism aims to introduce interoperability between the Application, Session (SIP protocol, [13]) and MAC layers, in addition to the control of the speech coding rate. Instead of the use of a transcoder for the return link, the mechanism assumes the existence of an Adaptive Multi-Rate (AMR) codec at the user end while the steps followed between the entities involved are illustrated in Fig. 1. The assumption is made that the satellite user attempts to make a call to the terrestrial network.

The cross-layer mechanism in this case is working in parallel to SIP in order to set-up the VoIP connection, according to immediately available resources, with the minimum delay (during the first four steps, as shown in Fig. 1). In parallel, it also requests additional $\mathrm{RBDC}$ capacity in order to maintain the highest possible VoIP rate. The mechanism is also interfacing to the $\mathrm{BoD}$ requester at the $\mathrm{MAC}$ layer, as it would have to alter the mechanism which normally requests capacity only based on the current size or traffic arrival rate of the queue. The estimation of available capacity and the reduction of coding mode is the same as the transcoding scenario, however this version is more suitable in the cases when:

- A low amount of CRA is allocated, therefore RBDC has to be requested. In this case the mechanism reduces the delay at call set-up;

- $\quad$ RBDC capacity is overbooked, and therefore the speech coding rate is adjusted in the case of RBDC shortage;

- A set of residential type of users is assumed, as the cost of transcoders is currently high.

\section{THE PREDICTIVE BOD ALGORITHM}

The Predictive $\mathrm{BoD}$ request algorithm is the second crosslayer mechanism proposed in this paper. It is designed in order to reduce TCP traffic delay induced by the requesting mechanism of $\mathrm{RBDC}$ or $\mathrm{A} / \mathrm{VBDC}$, which is assumed to be done via the allocation of periodic SYNC slots.

In order to estimate this delay, the guidelines of the DVBRCS standard [RD 16], in section 6.7.1 present examples for the segmentation of the return link capacity, where a terminal is allocated a SYNC slot with a period of 32 frames or $848 \mathrm{~ms}$ for the ATM profile assuming a $26.5 \mathrm{~ms}$ frame duration, while for the MPEG profile, depending on the terminal activity, this period can vary between $1.4-12 \mathrm{sec}$. The RTT for a GEO satellite is around $500 \mathrm{~ms}$ while the BoD controller scheduling latency is in the order of $50-100 \mathrm{~ms}$ according to the specific implementation. Finally, according to the standard, the latency between the terminal receiving the TBTP message and transmitting the burst assigned to it should not exceed $90 \mathrm{~ms}$. Therefore the total delay of the loop presented in Fig. 2 can be in the order of 2.5 seconds.

As shown in the same figure, a cross layer object at the satellite terminal, attempts to predict in the current SYNC slot the traffic which will be queued up and ready to transmit, until the next "opportunity" to request again as well as receive the requested capacity through the TBTP (Terminal Burst Time Plan). The information used to perform this estimation is the current state of the TCP Westwood congestion control window and Packet Error Probability statistics. The TCP Westwood related data is obtained from the transport layer which is assumed to use TCP Performance Enhancing Proxies at the edges of the satellite network and is used to emulate the evolution of the congestion window based on the calculation proposed in [14]. The latter is based on real time data on packets dropped from the network layer IP queues.

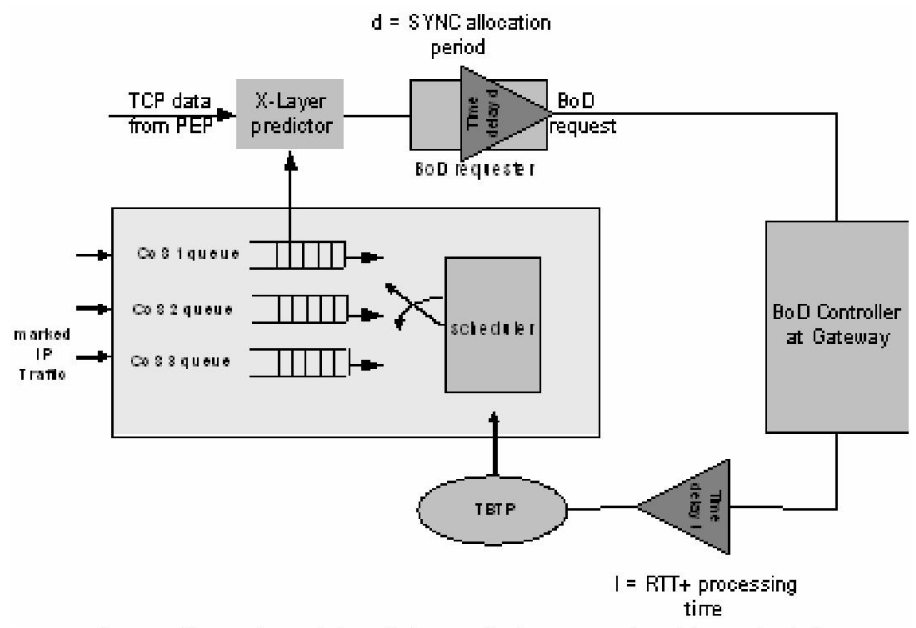

Fig. 2. Illustration of the of the Predictive $\mathrm{BoD}$ algorithm principle 
This prediction for RBDC and VBDC traffic respectively is the value of capacity requested from the $\mathrm{BoD}$ controller, assumed to reside at the Gateway.

Fig. 3 presents in a flowchart the algorithm implemented in the $\mathrm{BoD}$ controller in the GW, illustrating the way the X-layer predictor is called. As in the reference architecture, RBDC type of requests are used for $\mathrm{AF}$ type of traffic and $\mathrm{A} / \mathrm{VBDC}$ for $\mathrm{BE}$, two roots need to be configured in the PEP, controlled by two different congestion control windows. In addition, the AVBDC type of request is used to achieve synchronisation between the GW and RCST as to the amount of VBDC traffic requested and granted. Therefore, when the GW sets the VBDC empty traffic flag of the TBTP to one, if the terminal has some BE traffic still pending in its queue it will send a new VBDC request for it. Future simulations for this mechanism will provide results of the reduction in delay experienced by return link $\mathrm{AF}$ and $\mathrm{BE}$ TCP traffic at the RCST (EF traffic is assumed to make use of CRA, which is not requested).

\section{THE SIMULATOR}

In order to satisfy the modeling requirements of the crosslayer mechanisms, a simulator is needed to be able to model mechanisms and protocols across the whole protocol stack. Hence, ns, which is mainly used to simulate the network layer and above and SSP (System Simulation Platform), developed by EADS ASTRIUM for the modeling of the satellite and MAC layers have been modified to interoperate.

As a result a modular software platform that is able to simulate end-to-end applications across the whole protocol stack has been crated. In the integrated ns/SSP platform, packet simulation is shared between the two tools in the following way (assuming a forward link traffic flow as an example):

- Ns models the creation of the packets at the high layers of the sender and their circulation until the satellite

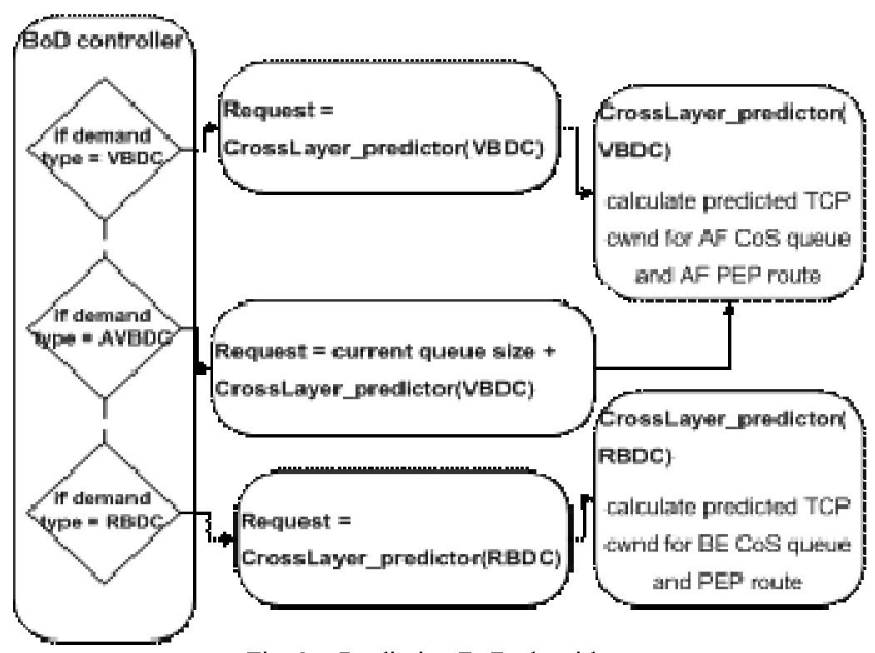

Fig. 3. Predictive BoD algorithm

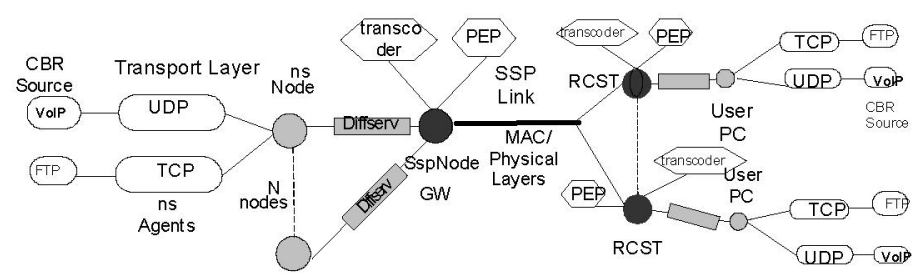

Fig. 4. The simulation topology in ns/SSP

gateway;

- SSP models the transmission of the packets between the gateway and the satellite terminal according to the DVB-S2/RCS standards, by encapsulating ns packets into SSP traffic cells;

- Ns models the transmission of the packets from the satellite terminal to the final destination.

This principle is further illustrated in Fig. 4.

\section{A. Initial simulation results}

Examples of simulation results using the ns/ssp simulator are presented in this paper. The simulation topology is illustrated in Fig. 4 while some variable simulation parameters are presented in TABLE I. Using the ns/SSP tool, specific applications, transport and network layer protocols can be modeled at the ns domain allowing the study of their characteristics. For the satellite system nodes (SspNode), protocols up to the network layer are modeled in ns, while then packets enter a simulated multibeam satellite system in SSP where mechanisms such as Call Admission Control, Bandwidth on Demand, Fade Mitigation Techniques as Adaptive Coding and Modulation or MPEG/ATM encapsulation are modeled. In addition congestion in the satellite network is controlled by loading the SSP simulator with background traffic and adjusting the number of active satellite terminals logged on per beam.

The DiffServ module developed in ns, as a type of link, also allows marking packets according to their source/destination as well as application they belong to. Finally, the PEP and transcoders are fully implemented in ns.

TABLE I

SIMULATION PARAMETERS SSP parameters (lower layers/satellite network)

\begin{tabular}{|l|c|}
\hline \multicolumn{2}{|c|}{$\begin{array}{c}\text { SSP parameters } \\
\text { (lower layers/satellite network) }\end{array}$} \\
\hline Number of beams & 1 (up to 10 can be used) \\
\hline Number of RCSTs per beam & 1000 (variable) \\
\hline Forward TDM rate & $43-116 \mathrm{Mbps}$ \\
\hline Return link rate per RCST & $240-480 \mathrm{kbps}$ \\
\hline RCST min. guaranteed data rate & $32 \mathrm{kbps}$ \\
\hline \multicolumn{2}{|c|}{ NS parameters } \\
\hline \multicolumn{2}{|c|}{ (upper layers/terrestrial network) } \\
\hline End-user VoIP speech coding & $23.85 \mathrm{kbps}$ \\
\hline Number of RCSTs (ns nodes) & 2 \\
\hline End user TCP & New Reno \\
\hline Satellite Link TCP (used in & Vegas \\
\hline
\end{tabular}




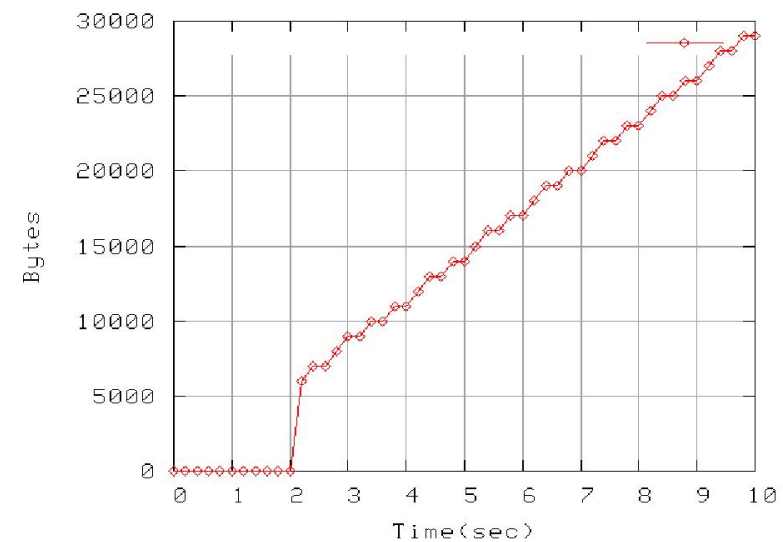

Fig. 5. Simulation results at ns level

Fig. 5 and Fig. 6 provide examples of simulation results at higher and lower layers for the current reference architecture. In Fig. 5, the reception of VoIP packets is monitored at the transport layer of the receiver, showing a 2 sec delay between call set-up and reception of data. In Fig. 6, a graph of the TBTP is presented, obtained by the SSP simulator, which plots the used slots across different carriers due to DRA. This feature as well as monitoring several other parameters at MAC layer facilitates the assessment of DAMA algorithms.

\section{CONCLUUSIONS}

This paper has illustrated the concept of cross-layer design for satellite networks, as well as presented a novel simulator, integrating a multibeam satellite system MAC/physical layer simulator within the ns-2 popular tool.

The two mechanisms proposed introduce interactions between attached and non attached layers of the protocol stack in order to improve VoIP user perceived quality as well as reduce the delay of TCP flows for the satellite return link.

Future work will target to enhance the algorithms using alternative information even from different layers as well as provide simulation results for the assessment of the two mechanisms in comparison to the reference architecture.

\section{REFERENCES}

[1] M. van der Schaar, S. Shankar, "Cross-layer Wireless Multimedia Transmission: Challenges, Principles and New Paradigms", IEEE Wireless Communications Magazine, 2005.

[2] M. V. Schaar, M. Tekalp, "Integrated Multi-Objective Cross-Layer Optimization for Wireless Multimedia Transmission" IEEE Wireless communication magazine, 2005.

[3] Chini, G. Giambene et al., "Dynamic Resource Allocation based on a TCPMAC Cross-Layer Approach for Interactive Satellite Networks", 2nd International Symposium on Wireless Communication Systems, September 2005, pages: $657-661$.

[4] N. Celandroni, F. Davoli et al., "An Overview of Some Techniques for Cross-Layer Bandwidth Management in Multi-Service Satellite IP

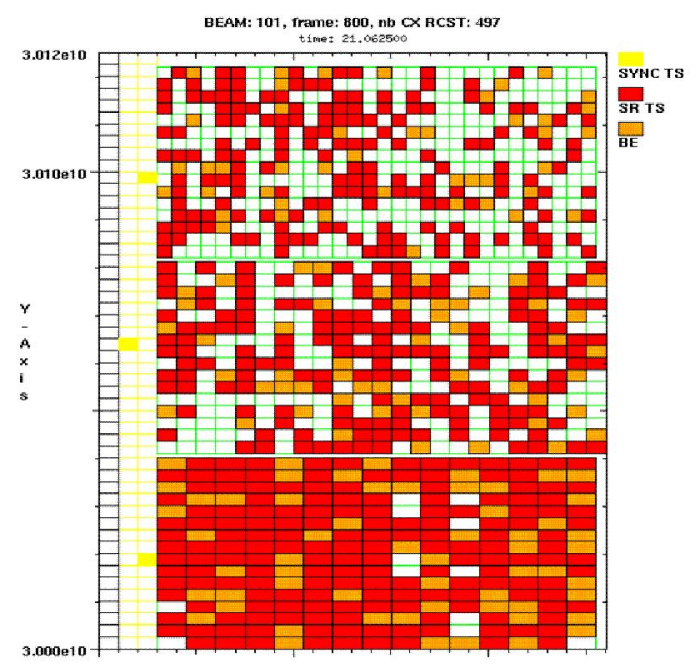

Fig. 5. Simulation results at SSP level

Networks", Workshop on Advances in Satellite Communications: New Services and Systems, IEEE Globecom '05, St. Louis, MO, Dec. 2005.

[5] N. Celandroni, F. Davoli et. al., "Long-Lived TCP Connections via Satellite: Cross-Layer Bandwidth Allocation, Pricing and Adaptive Control", IEEE/ACM Transactions on Networking (TON), 2006.

[6] H. Skinnemoen, A. Vermesan et al., "VoIP over DVB-RCS with QoS and Bandwidth on Demand", IEEE Wireless Communications, October 2005.

[7] M. Lambert, "VoIP over Satellite, An EMS Technologies Canada Technical Notes", May 2004, Revision 1-2.

[8] A. Vermesan, H. Skinnemoen et al., "VoIP over DVB-RCS, A Radio Resource and QoS Management Perspective", VoIP White Paper.

[9] H. Skinnemoen, A. Vermesan et al., "Voice over IP over Satellite with Applications to DVB-RCS, BGAN and iPSTAR Broadband Satellite Systems", $10^{\text {th }} \mathrm{Ka}$-band and Broadband Communication Conference, Vicenza, Italy, 30 Sept. -2 Oct., 2004.

[10] Z. Qiao, L. Sun, "A new method for VoIP Quality of Service control using combined adaptive sender rate and priority marking", IEEE Communications Society, Issue 0-7803-8533-0/04, 2004.

[11] K. Homayounfar, "Rate Adaptive Speech Coding for Universal Multimedia Access", IEEE Signal Processing Magazine, Volume 20, March 2003.

[12] J. Rosenberg. H. Schulzrinne et al., "SIP: Session Initiation Protocol", RFC 3261, June 2002.

[13] A. Zanella, G. Procissi et al., "TCP Westwood: Analytic Model and Performance Evaluation", Global Telecommunications Conference, 2001 (GLOBECOM'01).

[14] L. Chisci, R. Fantacci, T. Pecorella, "Predictive Bandwidth Control for GEO Satellite Networks", Proc. IEEE Internat. Conf. Commun. (ICC 2004).

[15] ETSI TR 101 790, "Digital Video Broadcasting (DVB); Interaction channel for Satellite Distribution Systems; Guidelines for the use of EN 301790 ", March 2005.

[16] M. Kalama, M-P. Kluth, S. Mourgues et el., Cross-Layer design for TCP applications and VoIP over satellite, to appear in Proc. $12^{\text {th }}$ Ka-band and Broadband Communication Conference, Napoli, Italy, September 2006.

[17] The Network Simulator ns-2: Documentation, http://www.isi.edu/nsnam/ns/ns-documentation.html.

[18] ETSI EN 301790 V1.4.1, Digital Video Broadcasting (DVB); Interaction channel for satellite distribution systems, March 2009.

[19] ETSI EN 302307 V1.1.1, Digital Video Broadcasting (DVB); Second generation framing structure, channel coding and modulation systems for Broadcasting, Interactive Services, News Gathering and other broadband satellite applications, March 2005. 